\title{
A Study to Evaluate the Effects of Relaxium in Subjects with Insomnia
}

\author{
Adams $\mathrm{T}^{1}$ and Duchin $\mathrm{K}^{{ }^{2}}$ \\ ${ }^{1}$ Center for Wellness, Fort Lauderdale, FL, USA \\ ${ }^{2}$ Principal Consultant, KLD Associates, USA
}

*Corresponding author: Duchin K, Principal Consultant, KLD Associates, USA, Tel: +1-408-694-7548, E-mail: kduchin@gmail.com

Citation: Adams T, Duchin K (2021) A Study to Evaluate the Effects of Relaxium in Subjects with Insomnia. J Insomn Sleep Disord 1(1): 103. doi: 10.15744/jisd.2021.1.103

Received Date: February 17, 2021 Accepted Date: March 01, 2021 Published Date: March 03, 2021

\begin{abstract}
Sleep disorders, which include insomnia are conditions that affect the onset, duration and quality of sleep, and as a result, can interfere with an individual's normal physical, mental, and emotional well-being. The primary objective of this study was to investigate the effect of Relaxium, an all-natural sleep aid, administered prior to bedtime on sleep parameters, compared to placebo, in subjects with insomnia. This was a double-blind placebo-controlled study with a 1-week placebo lead in period, followed by a 14-day period of either Relaxium or placebo (1:1). The main outcome measures were changes in Leeds Sleep Evaluation Questionnaire (LSEQ), daily diary and wrist actigraphy. Self-assessments for LSEQ and diary data were made from baseline to end of study. Based on the LSEQ, Relaxium treatment resulted in improved 1) quality of sleep, 2) getting to sleep, 3) behavior following awakening and 4) awakening following sleep, compared to placebo. The treatment differences between Relaxium on the 100-mm Visual Analog Scale (VAS) for these variables ranged from 10.2 to $12.3 \mathrm{~mm}$ (all $\mathrm{p}<0.034$ ). For the daily diary, Relaxium resulted in improvement in daytime concentration. The number of days that subjects reported no difficulty in concentration was $21 \%$ and $38 \%$ for placebo and Relaxium ( $p=0.013$ ), respectively. Overall, Relaxium treatment improved various parameters related to sleep, which included easier time to fall asleep, calmer sleep with less wakeful periods, easier awakening following sleep, and improved alertness after awakening. Subjects had less difficulty in concentration during the day, compared to placebo. Wrist actigraphy did not show any differences between treatments for time in bed, time asleep, time awake, light sleep, deep sleep and rapid eye movement (REM) sleep. No adverse events were reported in this study.
\end{abstract}

Keywords: Daily Dairy; Leeds Sleep Evaluation; Melatonin; Herbal Extracts; Insomnia

List of abbreviations: AFS: Awakening From Sleep; BFW: Behavior The Following Wakening; CDC: Centers for Disease Control and Prevention; CI: Confidence Interval; GABA: Gamma-Aminobutyric Acid; GTS: Getting to Sleep; IRB: Institutional Review Board; LSEQ: Leeds Sleep Evaluation Questionnaire; PSQI: Pittsburgh Sleep Quality Index; REM: Rapid Eye Movement; QoD: Daytime Diary; QoN: Nighttime Diary; QoS: Quality Of Sleep; VAS: Visual Analog Scale

\section{Introduction}

Insufficient rest or sleep is a prevalent problem for adults, with more than 50 million suffering from chronic sleep and wakefulness disorders and approximately 29\% reporting less than 7 hours of sleep per night in the United States. This is below the 7 to 9 hours of sleep recommended by the National Sleep Foundation $[1,2]$. Insufficient rest or sleep may be associated with chronic diseases, mental disorders, health-risk behaviors, limitations of daily functioning, injury and mortality [2]. In a CDC report analyzing responses via the Behavioral Risk Factor Surveillance System more than 35\% of respondents reported unintentionally falling asleep during the day and nodding off and falling asleep while driving was reported in $4.7 \%$ of respondents further indicating the dangerous impact of insufficient sleep [2].

A novel formulation of various herbs and known sleep inducers has been developed and is marketed in the United States under the tradename Relaxium. Key ingredients include melatonin, L-tryptophan, gamma-aminobutyric acid (GABA) and several herbal extracts (e.g., Sensoril ${ }^{\oplus}$, Ashwagandha, Valerest ${ }^{\mathrm{TM}}$, a blend of hops and valerian and Chamomile Passionflower). Many herbs like Valerest ${ }^{\mathrm{TM}}$ and Chamomile Passionflower have a long history of use as mild sedatives and hypnotics which may contribute to sleep improvement [3-8]. Extensive studies have been conducted on immediate release and sustained release melatonin products [9]. A recent meta-analysis of 19 studies involving 1,683 subjects, demonstrated that melatonin significantly improves sleep in subjects with primary sleep disorders compared to placebo. Melatonin reduces sleep-onset latency, increases total sleep time and improves overall sleep quality compared to placebo to a statistically significant degree. 


\section{Materials and Methods}

This was a double blind, repeated dose study in subjects with insomnia and was conducted from July 2019 to January 2020 . Subjects were recruited from local physicians and from social media advertising. Prior to enrollment, subjects completed the Pittsburgh Sleep Quality Index (PSQI). This is a validated measure of sleep quality that differentiates "poor" from "good" sleep by measuring 7 areas: subjective sleep quality, sleep latency, sleep duration, habitual sleep efficiency, sleep disturbances, use of sleeping medication and daytime dysfunction.

The study consisted of a 1-week lead-in period with placebo and the subjects were to complete daily sleep diaries [each morning for the quality of their sleep (QoN) for the previous night, each evening the overall quality of day (QoD), including levels of daytime energy and concentration] all on a 5-point scale. Wrist actigraphy (Fitbit Inspire) during sleep was used to assess quality and sleep duration over this period. Subjects also completed the LSEQ that measured four domains of sleep and morning behavior: Ease of getting to sleep (GTS), quality of sleep (QoS), hangover on awakening from sleep (AFS) and alertness and behavioral integrity the following morning (BFW) using a VAS. If subjects were compliant with these tasks, they were randomized in a double-blind manner to Relaxium or placebo once daily taken about 1 hour before bedtime for 2 weeks. During this Treatment Phase, daily sleep diaries were to be completed and wrist actigraphy were to be worn daily. The LSEQ was to be completed on Day 5, 6 and 7 (end of the lead-in period) and on Days 8,12,13,14 and 15 in the Treatment Phase. Subjects were seen in the clinic at the end of Weeks 1 and 2 of the double-blind treatment period.

\section{Inclusion Criteria}

Prospective subjects (20 to 80 years) with insomnia for at least one month, not related to other medical conditions, were enrolled. Other inclusion criteria were self-reported PSQI questionnaire score of 5 or higher and general good health without evidence of clinically significant disease as determined by medical history and urine drug screens. At screening, subjects had to agree to be willing to complete the questionnaires and diaries and adhere to a regular sleep routine with similar bedtime and awakening times.

\section{Exclusion Criteria}

Subjects with clinically unstable medical abnormality, chronic disease or history or presence of significant neurological disorders (including cognitive disorders), depression, schizophrenia, anxiety disorder, dementia, chronic pain, frequent nightly urination (>2 times per night), seizure disorder, restless leg syndrome, diagnosis of sleep apnea or risk factors for undiagnosed sleep apnea (witnessed apneic episodes) and/or use of psychotropic medication or beta blockers were excluded. Other exclusion criteria included all concomitant medications that are known to affect sleep or those known to interfere with drug metabolizing enzymes activity within 14 and 28 days prior to Day 1, respectively, as well as during the study. A history of alcoholism, drug addiction or participation in a clinical trial within the past year, or 30 days of entering the study, was prohibited.

Participants provided written informed consent and received instructions on how to complete the daily sleep diaries, use of the wrist actigraphy and completion of the LSEQ on pre-specified days during the study. If subjects met the criteria, they were given a one-week supply of placebo capsules and assigned a subject number. If they were compliant with completion of the diaries, the LSEQ and wrist actigraphy, they were randomized in a double-blind manner to either Relaxium or placebo. A 2-week supply of blinded medication was given to the subject. The Institutional Review Board (Integreview IRB) approved the study and all advertisements.

\section{Efficacy Analyses}

The sleep quality from the diary, LSEQ and wrist actigraphy that were collected during the run-in period (Week 1) served as the baseline. Data collected from Days 8 to 15 (Week 2 double blind period) were used to evaluate treatment effect. The diary individual values for each question were scored on a 5-point scale for the quality of their sleep, the overall quality of day, including levels of daytime energy and concentration.

For LSEQ, the subject drew a vertical mark on the line for each response. The distance was measured from left to right for each of the 10 queries. Lower values indicated poor sleep and higher numbers represented better sleep. The numbers of questions for getting to sleep domain, quality of sleep domain, awake following sleep domain and behavior following sleep domain were 3, 2, 2 and 3, respectively. Within each domain, the average of the 2 or 3 scores was generated, and that value was used in the analysis.

For wrist actigraphy, data from the lead-in period were averaged as a baseline and compared to the data from Days 8-14. Variables measured were minutes in bed and total sleep time (total minutes in light sleep, deep sleep and REM sleep) while in bed.

\section{Data Analysis for Sleep}

Descriptive statistics were calculated for all variables (one value for the entire treatment period) and included group means and $95 \%$ confidence intervals. 
Sleep quality from the daily diary and LSEQ data collected during the run-in period were used to evaluate the baseline severity and data collected from Days 8 to 15 were used to evaluate treatment effect. To assess subject changes in sleep, for each of the LSEQ domains and the daily diary, repeated measures analysis of the variance test was performed to evaluate the treatment differences between placebo and Relaxium from baseline (end of Week 1) to the end of Week 2 (end of the double-blind period) were compared. For the LSEQ analysis, subjects needed to have 3 measurements for each of the domains. For the daily diary data, subjects needed to have 6 responses for each question.

The Fishers Exact Test was used to determine if there were differences in the number of days in which there was no difficulty in concentration. For all analyses, statistically significant differences were declared when $\mathrm{p} \leq 0.05$.

\section{Results}

\section{Demography}

A total of 38 subjects enrolled in the study and 37 completed the lead in period and were randomized to either placebo ( $\mathrm{n}=17)$ or Relaxium $(n=20)$. One subject from each group did not complete the study. The mean $( \pm S D)$ ages in the Relaxium $(55.6 \pm 30$ years) and placebo ( $52.1 \pm 26$ years) groups were similar. Females comprised $76.9 \%$ and $63.1 \%$ of the population in the placebo and Relaxium groups, respectively. In the placebo group $75 \%$ of subjects were white and in the Relaxium group, the $68.8 \%$ of subjects were also white. The mean PSQI scores were similar (13.8 placebo vs. 14.1 Relaxium) between the treatment groups.

\section{Daily Diary}

Responses to each question in the diary were averaged for each subject in the lead-in and double-blind portion. In both groups, 1 subject did not complete the diary during the lead-in period, and 2 subjects in each group did not complete the diary data during the blinded portion of the study. Most subjects completed 6 to 7 diaries per week.

The mean (95\% Confidence Interval or CI) rating values for each of the 4 parameters of sleep for the baseline and treatment periods for subjects, beginning with 6 days of baseline through the second week of treatment are shown in Table 1. Baseline values were similar between the groups.

\begin{tabular}{|c|c|c|c|c|}
\hline \multirow{2}{*}{ Variable } & \multicolumn{2}{|c|}{ Placebo $(\mathrm{N}=17)$} & \multicolumn{2}{|c|}{ Relaxium (N=20) } \\
\hline & Baseline & Blinded & Baseline & Blinded \\
\hline \multicolumn{5}{|c|}{ QoN quality of sleep the previous night $(1=$ very poor, $5=$ very good $)$} \\
\hline Mean & 2.895 & 3.406 & 2.93 & 3.29 \\
\hline $95 \% \mathrm{CI}$ & $2.500,3.291$ & $3.011,3.801$ & $2.567,3.297$. & $2.93,3.65$ \\
\hline $\mathrm{N}$ & 16 & 16 & 18 & 18 \\
\hline \multicolumn{5}{|c|}{ QoD Q1 overall quality of day $(1=$ very poor, $5=$ very good $)$} \\
\hline Mean & 3.125 & 3.600 & 3.111 & 3.528 \\
\hline $95 \% \mathrm{CI}$ & $\begin{array}{l}2.718 \\
3.5321\end{array}$ & $3.180,4.021$ & $2.651,3.571$ & $3.067,3.988$ \\
\hline $\mathrm{N}$ & 16 & 16 & 18 & 18 \\
\hline \multicolumn{5}{|c|}{ QoD Q2 level of daytime energy ( $1=$ very poor, $5=$ very good $)$} \\
\hline Mean & 3.042 & 3.367 & 3.472 & 3.426 \\
\hline $95 \% \mathrm{CI}$ & $2.691,3.517$ & $2.94,3.79$ & $3.029,3.869$ & $2.983,3.864$ \\
\hline $\mathrm{N}$ & 16 & 16 & 18 & 18 \\
\hline \multicolumn{5}{|c|}{$\begin{array}{l}\text { QoD Q3 level of difficulty in concentration }(0=\text { no difficulty, } 1=\text { mild, } \\
2=\text { moderate, } 3=\text { severe , } 4=\text { very severe difficulty })\end{array}$} \\
\hline Mean & 1.625 & 1.287 & 1.278 & 0.981 \\
\hline $95 \% \mathrm{CI}$ & $1.305,1.945$ & $0.958,1.620$ & $\begin{array}{c}0.8445 \\
1.712\end{array}$ & $0.547,1.416$ \\
\hline $\mathrm{N}$ & 16 & 16 & 18 & 18 \\
\hline
\end{tabular}

\begin{tabular}{|c|c|c|c|c|c|c|c|}
\hline QoN & P-value & QoD Q1 & P-value & QoD Q2 & P-value & QoD Q3 & P-value \\
\hline-0.36 & 0.1621 & -0.44 & 0.0889 & -0.53 & 0.0584 & 0.79 & 0.0021 \\
\hline \\
QoD -daytime diary, QoN = nighttime diary \\
Table 2: Mean treatment differences in responses to the four \\
questions in the daily diary between Relaxium and Placebo Groups
\end{tabular}


Changes from baseline to the end of Week 2 were evaluated and the treatment differences in the daily scores between Relaxium and placebo groups are shown in Table 2. For subjects with complete data for both treatment arms there was a statistically significant difference in concentration levels (QoD Q3) between the 2 treatment groups. None of the other outcome measures were statistically significant although there was a trend for improvement in daytime energy with Relaxium.

Consistent with the finding for differences in difficulty in concentration, while taking blinded treatment, the number of days that subjects reported no difficulty in concentration was 19 of 90 days for placebo (21\%) and 41 of 108 days for Relaxium treatment (38\%). The difference between the 2 groups was highly statistically significant at $\mathrm{p}=0.013$. Thus, there was about an $80 \%$ increase in the number of days the subjects reported no difficulty in concentration compared to placebo.

\section{Leeds Sleep Evaluation Questionnaire}

Subjects completed LSEQ over the last 3 to 4 days of each treatment period. Higher values indicate improved sleep and better outcomes after awakening. Baseline values for AFS and BFW were similar between the two groups, whereas the values for GTS (36.4 mm, placebo vs. $29.0 \mathrm{~mm}$ Relaxium) and QoS (36.5 mm, placebo vs. $22.2 \mathrm{~mm}$ Relaxium), were lower in the Relaxium group indicating that those subjects randomized to Relaxium reported more difficulty getting to sleep and a lower quality of sleep.

For the repeated measures analysis of variance, only subjects with complete data for both treatment periods were included (Table 3 ). Compared to baseline, Relaxium treatment resulted in improved parameters such as getting to sleep (easier time to fall asleep, less time to fall asleep and more sleepy than normal) and quality of sleep (calmer sleep with fewer wakeful periods). For behavior and alertness following wakening, subjects in both groups felt they were more alert after awakening and awakening was easier from the lead-in period, but the effects were greater in the Relaxium group (Tables 3 and 4 ).

\begin{tabular}{|c|c|c|c|c|}
\hline \multirow{2}{*}{ Variable } & \multicolumn{2}{|c|}{ Placebo $(\mathrm{N}=15)$} & \multicolumn{2}{|c|}{ Relaxium $(\mathrm{N}=14)$} \\
\hline & Baseline & Blinded & Baseline & Blinded \\
\hline \multicolumn{5}{|c|}{ GTS (getting to sleep, compared to usual) } \\
\hline Mean & 35.80 & 44.17 & 27.92 & 48.01 \\
\hline $95 \% \mathrm{CI}$ & $\begin{array}{l}27.92 \\
43.66\end{array}$ & $\begin{array}{l}36.291 \\
52.043\end{array}$ & $\begin{array}{l}20.69 \\
35.15\end{array}$ & $\begin{array}{c}40.778 \\
55.25\end{array}$ \\
\hline $\mathrm{N}$ & 15 & 15 & 14 & 14 \\
\hline P value & & 0.13 & & 0.0004 \\
\hline \multicolumn{5}{|c|}{ QoS (quality of sleep, compared to normal sleep) } \\
\hline Mean & 35.84 & 44.21 & 22.56 & 53.214 \\
\hline $95 \% \mathrm{CI}$ & $\begin{array}{l}26.40 \\
45.28\end{array}$ & $\begin{array}{l}34.77 \\
53.65\end{array}$ & $\begin{array}{l}12.18 \\
32.94\end{array}$ & $\begin{array}{l}43.39 \\
63.05\end{array}$ \\
\hline $\mathrm{N}$ & 15 & 15 & 14 & 14 \\
\hline P value & & 0.21 & & 0.002 \\
\hline \multicolumn{5}{|c|}{ AFS (awakening following sleep, compared to usual) } \\
\hline Mean & 34.83 & 48.11 & 34.92 & 58.595 \\
\hline $95 \% \mathrm{CI}$ & $\begin{array}{l}25.46 \\
44.21\end{array}$ & $\begin{array}{l}38.74 \\
57.48\end{array}$ & $\begin{array}{l}25.68 \\
44.15\end{array}$ & $\begin{array}{l}49.80 \\
67.39\end{array}$ \\
\hline $\mathrm{N}$ & 15 & 15 & 14 & 14 \\
\hline P value & & 0.05 & & 0.001 \\
\hline \multicolumn{5}{|c|}{$\begin{array}{l}\text { BFW (behavior following awakening how you feel } \\
\text { when you wake up) }\end{array}$} \\
\hline Mean & 35.40 & 50.24 & 31.89 & 56.91 \\
\hline $95 \%$ CI & $\begin{array}{l}26.68 \\
49.11\end{array}$ & $\begin{array}{l}41.52 \\
58.95\end{array}$ & $\begin{array}{l}23.46 \\
40.33\end{array}$ & $\begin{array}{l}48.67 \\
65.15\end{array}$ \\
\hline $\mathrm{N}$ & 15 & 15 & 14 & 14 \\
\hline P value & & 0.02 & & 0.002 \\
\hline
\end{tabular}

$\mathrm{P}$ value based on the within group differences between Baseline and Blinded periods.

Table 3: Mean (95\% CI) VAS Scores ( $\mathrm{mm}$ ) for LSEQ (Subjects with both Baseline and Blinded Treatment Periods)

\begin{tabular}{|c|c|c|c|c|}
\hline \multirow{2}{*}{ Difference between Relaxium and Placebo Groups $(\mathbf{m m})$} & QoS & GTS & BFW & AFS \\
\cline { 2 - 5 } & +12.3 & +12.2 & +10.2 & +10.4 \\
\hline P-value & 0.002 & 0.006 & 0.028 & 0.034 \\
\hline
\end{tabular}

AFS = awakening following sleep (compared to usual), BFW = behavior following awakening (how you feel when you wake up), GTS = getting to sleep (compared to usual), QoS = quality of sleep (compared to normal sleep)

Table 4: Change from Baseline - Mean Treatment Differences Between Relaxium and Placebo 
For all 4 domains the treatment effect of Relaxium was statistically greater than for placebo. The mean differences for the treatments expressed as changes from baseline, are shown in Table 4.

To determine relative effectiveness, the mean changes in LSEQ from Week 1 to Week 2 were compared in subjects who had paired values. The ratios of the mean increase from baseline in VAS scores are shown in Table 5 and indicate that there was a 2.4 to 3.7fold more favorable effect in GTS and QoS, compared to baseline. The changes in AFS (easier time for awakening) and BFW (more alert) were also greater by 1.7 to 1.8 -fold.

\begin{tabular}{|c|c|c|c|}
\hline Parameter & Placebo $(\mathbf{m m})$ & Relaxium $(\mathbf{m m})$ & Fold Increase \\
\hline GTS & 8.37 & 20.09 & 2.4 \\
\hline QoS & 8.37 & 30.65 & 3.66 \\
\hline AFS & 13.28 & 23.67 & 1.78 \\
\hline BFW & 14.84 & 25.02 & 1.69 \\
\hline
\end{tabular}

AFS = awakening following sleep (compared to usual), BFW = behavior following awakening (how you feel when you wake up), GTS = getting to sleep (compared to usual), QoS = quality of sleep (compared to normal sleep) Table 5: Relative Effectiveness of Relaxium to Placebo

\section{Wrist Actigraphy}

Times were recorded for various sleep parameters (time in bed, time asleep, time awake, light sleep, deep sleep and REM sleep) were recorded for most of the subjects. For some subjects, not all measurements were taken for each of the days, or some of the variables could not be determined. The was likely due to either the device not being worn correctly or because of technical issues with the device. It does not appear that there is any difference for these sleep parameters between Relaxium and placebo.

\section{Safety}

There were no adverse events reported in this study. One subject was diabetic and took medication for that condition prior to and during the study. Other non-sleep related medications included lisinopril and L-Synthroid.

\section{Discussion}

Insomnia is a pathological condition caused by a variety of endogenous and exogenous factors. It is characterized primarily by the difficulty of initiating and maintaining sleep, which results in low-quality daily activity. With age, the prevalence of insomnia increases; one of the reasons for this is an involutional decrease in the level of secretion of melatonin. According to epidemiological studies, $6 \%$ of adults in industrialized countries suffer from a chronic form of insomnia [10]. In addition to night manifestations, accompanied by an increase in sleep latency, a decrease in sleep time, low sleep efficiency and an increase in wakefulness during sleep, daytime manifestations of this disease are also formed. These may include fatigue, decreased short-term memory, decreased mood, headaches and gastrointestinal disturbances [11].

Based on the findings of this relatively small double-blind randomized placebo-controlled study, using a validated methodology, it was found that sleep was improved on the Relaxium treatment. The LSEQ findings showed that subjects had an easier time to fall asleep and the sleep was rated as calmer with less wakeful periods than during the lead-in period. In addition, awakening following sleep was easier and there was improved alertness after awakening.

Placebo treated subjects also reported the improvements in AFS and BFW, but the magnitude of the difference from the respective Lead in Period was significantly greater for Relaxium. The observation that placebo had some effect on AFS and BFW underscores the need to conduct double blind for evaluation of sleep.

Consistent with subjects being more alert, the findings from the daily diary found that concentration improved more significantly with Relaxium than placebo. The number of days that subjects reported no difficulty in concentration was about $80 \%$ higher with Relaxium compared to placebo.

These findings are clearly supportive of Relaxium's effects, but the contribution of each of the 6 sleep-inducing or anxiety/stress reducing components cannot be determined with this study. First melatonin, polysomnographic criteria for the effectiveness of insomnia therapy were achieved, namely, a decrease in sleep latency and an increase in the total sleep time and sleep efficiency $[12,13]$. Other components like Sensoril ${ }^{\circledR}$, a standardized extract of Ashwagandha (Withania somnifera), have been shown to lower serum cortisol [14] and is associated with improved sleep, although the assessment tools were not reported [15].

L-tryptophan, GABA and several herbal extracts (e.g., Valerest ${ }^{\mathrm{TM}}$, a blend of hops and valerian, Chamomile Passionflower) may have also contributed to Relaxium's effects. Salter and Brownie [16] reviewed 12 clinical trials (six of which were randomized, double blind, placebo-controlled trials) studied the effects of valerian on sleep parameters: total sleep time, sleep latency (i.e., time to fall asleep), slow wave sleep, nocturnal awakenings and sleep quality. Nine of these studies found valerian to be effective in 
improving at least one of the sleep parameters measured. However, it is important to note that five of these studies had significant methodological flaws, which limits the reliability of their findings and therefore the extent to which they can be applied to clinical practice. In addition, in most studies, valerian was administered for 1 day only, sample sizes ranged from 8 to 405 subjects and doses ranged from 300 to $950 \mathrm{mg} /$ day. A large scale, double blind, randomized, placebo-controlled trial found that 5.5\% [95\% CI $0.2-10.8]$ more participants in the valerian group perceived their sleep as better or much better $(\mathrm{p}=0.04)$ compared to the control (placebo) group [4].

Gamma-amino butyric acid is an amino acid contained in various foods and is known as an inhibitory transmitter of the central nervous system. Oral administration of GABA has effects on the autonomic nervous system which includes reducing anxiety [17] and stress [18] by increasing parasympathetic nerve activity [19]. Based on electroencephalograms, Yamatsu et al. [20] demonstrated oral GABA (100 mg) administered for 1 week, 30 minutes before bedtime decreased sleep latency by 5.3 minutes in subjects with insomnia.

Zick and colleagues [21] conducted a randomized double-blind, placebo-controlled pilot trial of chamomile in 34 patients with primary insomnia for $\geq 6$ months. Patients were randomized to $270 \mathrm{mg}$ of chamomile twice daily or placebo for 28 days. Chamomile provided modest benefits of daytime functioning and mixed benefits on sleep diary measures relative to placebo in adults with chronic primary insomnia. Baek et al. [22] reported that passionflower was efficacious in at least one randomized clinical trial in healthy subjects.

Regarding L-tryptophan, doses of $1 \mathrm{~g}$ or more produces an increase in rated subjective sleepiness and a decrease in sleep latency. There are fewer firm data suggesting that L-tryptophan may have additional effects such as decrease in total wakefulness and/ or increase in sleep time. Best results (in terms of positive effects on sleep or sleepiness) have been found in subjects with mild insomnia, or in normal subjects reporting a longer-than-average sleep latency [23].

\section{Conclusion}

In summary, Relaxium treatment over 2 weeks had a positive impact on various sleep indices as shown using validated methodology.

\section{Declarations}

\section{Study Limitations}

Sample size, technical difficulties with wrist actigraphy, compliance with completion of diaries and questionnaires.

\section{Acknowledgments}

None

\section{Funding Source}

American Behavioral Research Institute

\section{Competing Interests}

None

\section{References}

1. McKnight-Eily LR, Liu Y, Perry GS, Presley-Cantrell LR, Strine TW, et al. (2008) Perceived Insufficient Rest of Sleep Among Adults-United States 2008. JAMA 302: 2532-39.

2. McKnight-Ely LR, Liu Y, Wheaton AG, Croft JB, Perry GS, et al. (2011) Unhealthy sleep related behaviors-12 states. MMWR 60: 233-8.

3. Bone K, Mills S (2000) Principles and Practice of Phytotherapy, Modern Herbal Medicine, Edinburgh: Churchill Livingstone, USA.

4. Oxman A, Flottorp S, Havelsrud K, Fretheim A, Odgaard-Jensen J, et al. (2007) A televised, web-based randomized trial of an herbal remedy (valerian) for insomnia. PLoS One 2: 1-10.

5. Donath F, Quispe S, Diefenbach K, Maure A, Fietze AI, et al. (2000) Critical evaluation of the effect of valerian extract on sleep structure and sleep quality. Pharmacopsychiatry 33: 47-53.

6. Dorn M (2000) Efficacy and tolerability of Baldrian versus oxazepam in non-organic and non-psychiatric insomniacs: a randomized, double-blind, clinical, comparative study. Forsch Komplementarmed Klass Naturheilkd 7.

7. Dressing H, Riemann D, Low H, Schred M, Reh C, et al. (1992) Insomnia: Are valeriana/melissa combinations of equal values to benzodiazepine? Therapiewoche 42: 726-36.

8. Kamm-Kohl AV, Jansen W, Brockmann P (1984) Modem valerian therapy of the nervous system. Medizinische Welt 35: 1450-4.

9. Ferracioli-Oda E, Qawasmi A, Bloch MH (2013) Meta-Analysis: Melatonin for the Treatment of Primary Sleep Disorders PLoS ONE 8: e63773.

10. Zhang W, Wing YK (2006) Sex differences in insomnia: A meta-analysis. Sleep 29: 85-93.

11. Edinger JD, Bonnet MH, Bootzin RR, Doghramji K, Dorsey CM, et al. (2004) Derivation of research diagnostic criteria for insomnia: Report of an American Academy of Sleep Medicine Work Group Sleep 27: 1567-96. 
12. Brzezinski A, Vangel MG, Wurtman RJ, Norrie G, Zhdanova I, et al. (2005) Effects of exogenous melatonin on sleep: A meta-analysis. Sleep Medicine Reviews 9: 41-5035.

13. Zakharov AV, Khivintseva EV, Pyatin VF, Sergeeva MS, Antipov OI (2019) Melatonin-Known and novel areas of clinical application. Neuroscience and Behavioral Physiology 49: 60-3.

14. Auddy B, Hazra J, Mitra A, Abedon B, Ghosal S (2008) A standardized withania somnifera extract significantly reduces stress-related parameters in chronically stressed humans: a double-blind, randomized, placebo-controlled study. JANA 11: 50-6.

15. Abedon B (2011) Sensori ${ }^{\circ}$ - "A Patented Extract that Reduces Stress and Enhances Sleep. NutraGenesis LLC, USA.

16. Salter S, Brownie S (2010) Treating Primary Insomnia. Aust Family Physician 39: 433.

17. Abdou AM, Higashiguchi S, Horie K, Kim M, Hatta H, et al. (2006) Relaxation and immunity enhancement effects of gamma-aminobutyric acid (GABA) administration in humans. Biofactors 26: 201-8.

18. Nakamura H, Takishima T, Kometani T, Yokogoshi H (2009) Psychological stress-reducing effect of chocolate enriched with $\gamma$-aminobutyric acid (GABA) in humans: assessment of stress using heart rate variability and salivary chromogranin A. Int J Food Sci Nutr 60 Suppl 5: 106-13.

19. Fujibasyashi M, Kamiya T, Takagaski K, Moritani T (2008) Activation of autonomic nervous system activity by the oral ingestion of GABA. J Japan Soc Nutr Food Sci 61: 129-33.

20. Yamatsu A, Yamashita Y, Maru I, Yang J, Tatsuzake J, et al.(2015) The improvement of sleep by oral intake of GABA and Apocynum venetum leaf extract. J Nutr Sci Vitaminol 61: 182-7.

21. Zick SM, Wright BD, Sen A, Todd J, Arnedt JT (2011) Preliminary examination of the efficacy and safety of a standardized chamomile extract for chronic primary insomnia: A randomized placebo-controlled pilot study. BMC Complementary and Alternative Medicine 11: 78.

22. Baek JH, Nierenberg AA, Gustavo Kinrys G (2014) Clinical applications of herbal medicines for anxiety and insomnia; targeting patients with bipolar disorder. N Z J Psychiatry 48: 705-15.

23. Hartmann E (1983) Effects of L-tryptophan on sleepiness and on sleep. J Psychiatry Res 17: 107-13.

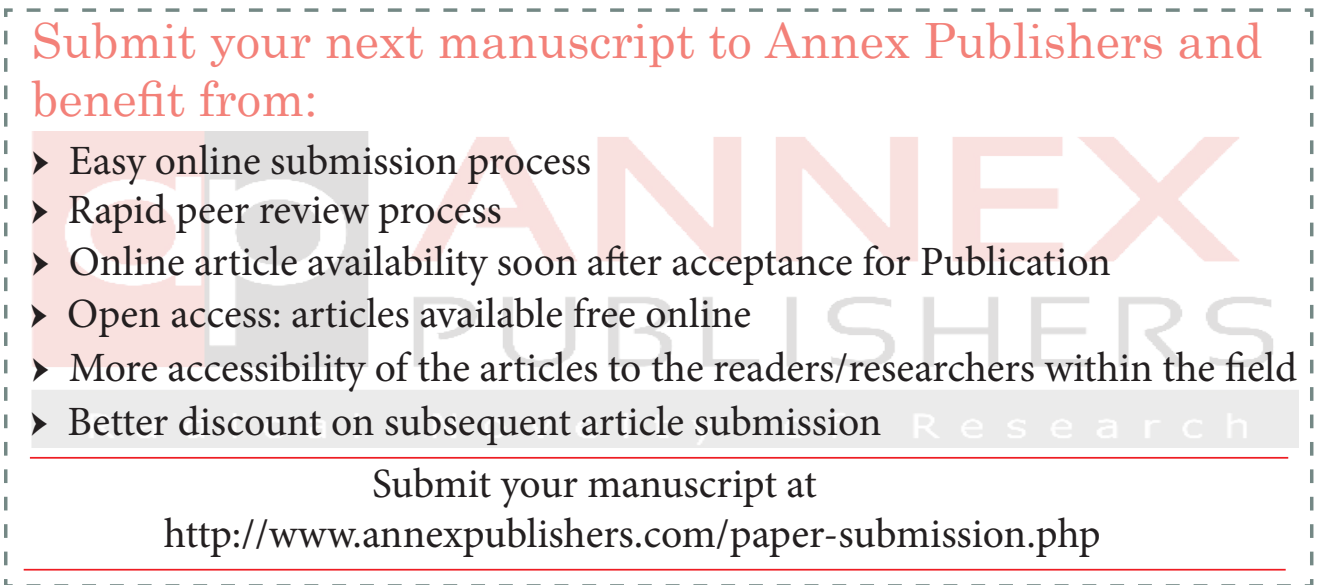

